\title{
What Has the COVID-19 Pandemic Taught Us so Far? Addressing the Problem from a Hepatologist's Perspective
}

\author{
Nahum Méndez-Sánchez*1,2 , Alejandro Valencia-Rodríguez ${ }^{1}$, Xingshun $i^{3}$, Eric M. Yoshida ${ }^{4}$, \\ Manuel Romero-Gómez ${ }^{5}$, Jacob George ${ }^{6}$, Mohammed Eslam ${ }^{6}$, Ludovico Abenavoli ${ }^{7}$, \\ Weifen $\mathrm{Xie}^{8}$, Rolf Teschke ${ }^{9}$, Andres F. Carrion ${ }^{10}$ and Andrew P. Keaveny ${ }^{11}$
}

\begin{abstract}
${ }^{1}$ Liver Research Unit, Medica Sur Clinic \& Foundation, Mexico City, Mexico; ${ }^{2}$ Faculty of Medicine, National Autonomous University of Mexico, Mexico City, Mexico; ${ }^{3}$ Department of Gastroenterology, General Hospital of Northern Theater Command (Formerly General Hospital of Shenyang Military Area), Shenyang, China; ${ }^{4}$ Division of Gastroenterology, University of British Columbia, Vancouver, BC, Canada; ${ }^{5}$ UCM Digestive Diseases and CIBERehd, Institute of Biomedicine of Seville (IBiS), SeLiver Group, Virgen del Rocío University Hospital, University of Seville, Seville, Spain; ${ }^{6}$ Storr Liver Centre, Westmead Institute for Medical Research, Westmead Hospital and University of Sydney, Sydney, NSW, Australia; ${ }^{7}$ Department of Health Sciences, University Magna Graecia of Catanzaro, Italy; ${ }^{8}$ Department of Gastroenterology, Changzheng Hospital of the Second Military Medical University, Shanghai, China; ${ }^{9}$ Department of Internal Medicine II, Division of Gastroenterology and Hepatology, Klinikum Hanau, Academic Teaching Hospital of the Medical Faculty, Goethe University Frankfurt/Main, Hanau, Germany; ${ }^{10}$ Division of Gastroenterology and Hepatology, University of Miami, Miller School of Medicine, Miami, FL, USA; ${ }^{11}$ Department of Transplantation, Mayo Clinic, Jacksonville, Florida, USA
\end{abstract}

Citation of this article: Méndez-Sánchez $N$, Valencia-Rodríguez A, Qi X, Yoshida EM, Romero-Gómez M, George J, et al. What has the COVID-19 pandemic taught us so far? Addressing the problem from a hepatologist's perspective. J Clin Transl Hepatol 2020;8(2):109-112. doi: 10.14218/JCTH.2020.00024.

As of today, March 30, 2020, when this Editorial is being written, the severe acute respiratory syndrome coronavirus-2 (SARS-CoV-2), causal agent of the coronavirus disease (COVID-19) has been confirmed in more than 745,000 cases worldwide and has claimed the lives of more than 35,000 people. ${ }^{1}$ In addition to the morbidity and mortality associated with COVID-19, this betacoronavirus has placed several of the world's major economies in strife, mainly in Western Europe and North America, paralyzing travel and regular social interactions, making COVID-19 undoubtedly one of the most important pandemics in human history.

While we are in the midst of battling this pandemic, we have already learned some lessons from a cruel teacher: a) the importance of a strong association that must exist between governments and the scientific community in implementing a broad range of measures to contain and, in the future, prevent this type of epidemic; b) the potential for this pandemic to indirectly, due to less available resources, increase liver-related outcomes morbidity and mortality, including liver transplantation; c) the necessity to develop

\footnotetext{
Abbreviations: ACE2, angiotensin-converting enzyme-2 receptor; ALT, alanine aminotransferase; AST, aspartate aminotransferase; BCG, Bacillus CalmetteGuerin; COVID-19, coronavirus disease; HLA, human leukocyte antigen; NCP, COVID-19 pneumonia; S, Spike; SARS-CoV-2, severe acute respiratory syndrome coronavirus-2.

Received: 4 April 2020; Accepted: 5 April 2020

* Correspondence to: Nahum Méndez-Sánchez, Liver Research Unit, Medica Sur Clinic and Foundation, National Autonomous University of Mexico, Puente de Piedra 150, Col. Toriello Guerra, ZP 14050, México City, México. Tel: +52555424-4629, E-mails: nmendez@medicasur.org.mx; nah@unam.mx
}

new working practices in multidisciplinary teams that will provide appropriate levels of care for patients from intensive care units to the outpatient setting. COVID-19 may make virtual clinic visits through telemedicine the norm and not the exception in some parts of the world. However, there are many questions that have yet to be answered. One of the most important to resolve is the understanding of the devastating impact of SARS-CoV-2 in specific geographic regions, such as Spain and Italy.

To better understand the current pandemic, we have to start by analyzing the mechanisms that COVID-19 possesses to infect humans and cause disease. We can observe the close similarity of SARS-CoV-2 with the SARS-CoV virus of 2002, that shares the same interaction between the viral protein Spike (commonly referred to as ' $S$ ') and the angiotensinconverting enzyme-2 receptor (ACE2) in the host. ${ }^{2}$ ACE2 is a protein with an important role in the regulation of cardiovascular, renal and liver function. It is highly expressed in the lungs and in other tissues, such as the liver (discussed later), intestine, and oral mucosa. ${ }^{3}$ Further, ACE2 is expressed more in Asian males compared to females and to other ethnic groups, possibly contributing to the susceptibility of Asians for developing respiratory infections by coronavirus species. ${ }^{4}$

Interestingly, in SARS-CoV there was a correlation between the susceptibility to infection of airway epithelia with the state of cell differentiation, and with ACE2 expression and location. SARS-CoV replication has been observed in polarized epithelia, exiting mainly in the apical zone. ${ }^{2}$ In this context, tobacco use is strongly associated with many lung diseases and cancer development. Although, strictly speaking no relationship has yet been proven to exist between COVID19 and tobacco use, smoking is well-recognized to upregulate the expression of the epidermal growth factor receptor in polarized airway epithelial cells. It may therefore increase the susceptibility to infection by SARS-CoV-2 by deregulating 
the ACE2 receptor. ${ }^{4}$ Considering that the European Region has the highest prevalence of tobacco use (29\%) worldwide, ${ }^{5}$ this may provide a possible explanation for the significant burden of COVID-19, as opposed to asymptomatic or mildly symptomatic infection seen in other parts of the world.

Moreover, the human genome is the basis for a large component of inter-individual phenotypic variability to disease. The discovery of the human leukocyte antigen (HLA) in the early 1970s clarified our understanding of the basis for many human diseases, especially in the field of infectious diseases, ${ }^{6}$ through a more well-founded comprehension of the interaction between environmental and host factors.

During the 2002 epidemic of SARS-CoV, some studies found an association between the severity of disease and the HLA-B*46:01 and HLA-B*07:03 alleles in Taiwanese and mainland Chinese populations, ${ }^{7,8}$ respectively. In contrast, expression of the HLA-DRB1*03:01 allele conferred protection against the disease in both Taiwanese and mainland Chinese populations. ${ }^{8,9}$ Surprisingly, a recent in silico analysis of viral peptide-HLA interaction that awaits publication suggests that the HLA-B*46:01 allele could also impact the severity of COVID-19 and that the HLA-B*15:03 allele could confer immunity to the disease. ${ }^{10}$

Vaccination policies between countries could be an important factor in susceptibility or protection against COVID-19. For example, it has been reported that the Bacillus CalmetteGuerin (commonly referred to as BCG) vaccine may confer protection against respiratory infections. Consistently, a recent study reported that countries that do not utilize the BCG vaccination routinely (Italy, the Netherlands, USA) have been most affected by COVID-19. ${ }^{11}$

From a hepatologist's perspective, COVID-19 must be of concern, especially since infection with this virus has led to complications in other organs. In the case of the liver, the first study conducted in patients diagnosed with COVID-19 pneumonia (NCP) found that 43 of the 99 patients developed a degree of liver injury characterized by an increase in aspartate aminotransferase (AST) and alanine aminotransferase (ALT) $;{ }^{12}$ one patient developed severe liver injury (ALT 7590 $\mathrm{U} / \mathrm{L}$, AST $1445 \mathrm{U} / \mathrm{L}$ ). In the latter case, acute liver injury secondary to hypoxic hepatitis could not be excluded. In the study, 97 patients (98\%) had a decrease in serum albumin and $75(76 \%)$ had increased levels of lactate dehydrogenase - a generalized systemic inflammatory response to the virus could explain these findings. Guan et al. ${ }^{13}$ carried out the largest study in NCP patients that included 1099 patients from 552 Chinese hospitals. They noted that patients with severe pneumonia were more likely to develop abnormal aminotransferase levels as compared with those with mild and moderate disease. A recent meta-analysis of four studies has also suggested an increased level of ALT is observed in $29 \%$ of patients with COVID-19. ${ }^{14}$ Yao et al. ${ }^{15}$ undertook a multiple regression analysis and suggested that the appearance of liver injury was probably related to the critical illness itself, with elevations of aminotransferases in the first week and hypoalbuminemia from the second week; both appear to be reversible with resolution of infection.

We anticipate a question that hepatologists will be asked is whether COVID-19 directly targets the liver?

It is a matter of debate whether COVID-19 is directly responsible for the development of liver injury, or whether the observed changes are secondary to the systemic inflammation triggered by infection (Fig. 1). The Chinese Digestion
Association of the Chinese Medical Doctor Association and the Chinese Society of Hepatology of the Chinese Medical Association recently stated that the development of liver injury in COVID-19 patients might be related to any of the following: 1) a direct hit from this virus; 2) systemic inflammation; 3) hepatic ischemia and hypoxia; 4) pre-existing liver disease; and 5) drug-related liver injury (especially the use of antibiotics or other hepatotoxic drugs in critically ill patients). ${ }^{16}$ As mentioned previously, ACE2 is expressed in other tissues, such as the liver. ${ }^{3}$ Interestingly, hepatocytes do not express the ACE2 receptor as much as bile duct epithelial cells, suggesting that, at least in theory, biliary tract could be more susceptible to SARS-CoV-2-driven injury. However, in the studies conducted so far, no increase in bile duct injury markers, such as gamma-glutamyl transferase and alkaline phosphatase, has been observed. Moreover, SARS-CoV-2 viremia seems to be uncommon, despite severe pneumonia with high viral replication in the lung. The virus has been detected in feces, even several days after clearance on nasopharyngeal swaps. Patients with more advanced liver diseases, including hepatitis $B$, and those with lower serum albumin levels showed impaired prognosis. A viral translocation from the gut to the liver could allow the virus to reach the liver and promote hepatic injury.

A recent mouse model of acute liver injury with partial hepatectomy found that at day 1 post-hepatectomy, hepatic expression of ACE2 in rats was downregulated. From day 3 , there was an up to two-fold increased expression of this receptor, with a normalization of values on day 7 , when the liver recovered and hepatocyte proliferation ceased. ${ }^{17}$ With this information, the authors proposed that the systemic inflammation derived from significant NCP could trigger liver necrosis and hepatocyte proliferation, upregulating the expression of ACE2 and therefore worsening liver injury. Similarly, liver biopsies from SARS-CoV patients showed a significant increase in mitotic cell numbers, with eosinophilic bodies and balloon-like hepatocytes, ${ }^{18}$ supporting a role for SARSCoV-2 in the development of liver injury.

Systemic inflammation and the subsequent multiple organ failure triggered by severe NCP would undoubtedly act synergistically in the development of liver injury. COVID-19 has been reported to upregulate the expression of proinflammatory cytokines, like IL-1 $\beta$, IL-6, and tumor necrosis factor-alpha. ${ }^{2}$ These cytokines are upregulated in a wide number of liver disorders, including viral hepatitis, metabolic associated fatty liver disease, and alcoholic liver disease. The use of antipyretics, such as paracetamol, and certain antivirals, like oseltamivir, lopinavir and ritonavir, for the management of COVID-19 has been widely reported to be associated with the development of liver injury. ${ }^{19}$

What precautions, then, should patients with chronic liver disease follow during this pandemic? Due to the exponential increase in cases of infection worldwide, it is very likely that gastroenterologists and hepatologists will encounter patients with chronic liver disease and concomitant COVID-19 infection.

In the case of viral hepatitis, there is still no reliable information that supports synergism with SARS-CoV-2. However, it is known that SARS-CoV patients with viral hepatitis are more prone to develop liver damage and severe hepatitis. This was likely due to enhanced replication of the hepatitis virus during SARS-CoV infection. ${ }^{20}$ In this context, some HLA class II haplotypes, like DRB1*1302, HLA-DR13, DQA1*0501, and DQB1*0301, have been related 
A Direct liver hit by the virus

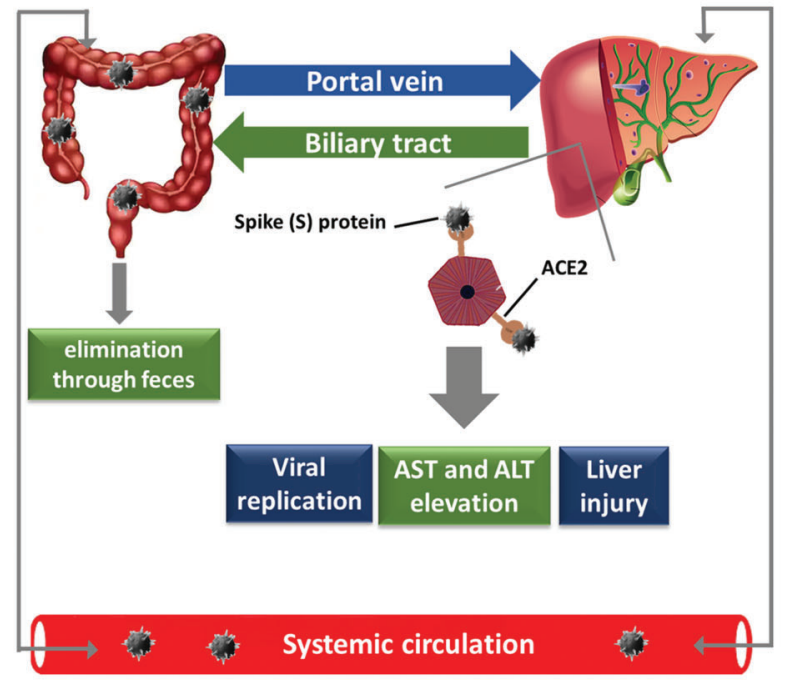

B Indirect causes of liver injury associated with COVID-19 infection

\section{Systemic inflammation}

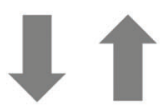

Liver ischemia and hypoxia

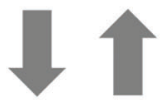

Pre-existing liver diseases

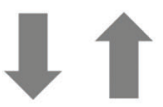

Drug-related liver injury

Fig. 1. Mechanisms of liver injury induced by SARS-CoV-2.

Abbreviations: ACE2, angiotensin converting enzyme 2; AST, aspartate aminotransferase; ALT, alanine aminotransferase.

to acute and chronic hepatitis B virus persistence. ${ }^{21}$ Thus, it would be useful to study risk-sharing alleles that might exist between viral hepatitis and COVID-19.

Patients with cholestatic diseases are another group that could present with more liver injury in the context of COVID-19 illness, as ACE2 is expressed in the epithelial cells of bile ducts. Moreover, immunocompromised states, such as those of individuals with cirrhosis and on immunosuppressive therapy, may place the patient at higher risk of severe illness. It has therefore been suggested that COVID-19 could accelerate the onset of complications in patients with compensated cirrhosis. ${ }^{22}$ To date, we are uncertain about the effects of immunosuppression in these patients and in post-transplant liver patients. During previous outbreaks of coronavirus infections, it was suggested that immunocompromised patients (adults and children) are not at increased risk of infection compared to the general population. ${ }^{23}$ However, if this pandemic has taught us anything, it is that the knowledge from previous experiences may not be generalizable to this new infection. A recent case report described a perioperative presentation of COVID-19 in a liver transplant recipient that jeopardized the graft. ${ }^{24}$ The groups of people who need to take extra precautions may expand as we learn more about SARS-CoV-2.

Even though there is no consensus in the management of these types of situations, an expert panel of physicians from Wuhan, China provided a list of suggestions in terms of precautions to follow in patients with chronic liver disease. ${ }^{25}$ They suggested avoiding home visits and going to crowded places, following a balanced diet, frequent handwashing of not less than $20 \mathrm{~s}$, as well as adequate ventilation and cleaning of the home. For inpatients, they suggested treatment by only one physician and a nurse, with strict handwashing and disinfection after any rounds or procedures, provision of adequate personal protective equipment, and establishing a clean care area.

We are experiencing the full impact of the SARS-CoV-2 pandemic on our countries, our profession, and our patients. As we meet this crisis head-on, the analysis of epidemiology data can provide a valuable tool to assess the current evolution of the COVID-19 outbreak worldwide and to evaluate the impact of the countermeasures adopted, leading to the development of appropriate healthcare policies, targeting precious resources and equipment to all the clinical situations we encounter. While we wait for advances in treating the virus and the development of a vaccine, changes in personal behavior and widely available testing for the virus could really make a difference in the outcomes for our patients and healthcare workers, while preventing further spread within our communities. In other words, "be safe and use common public health sense". We are all in this crisis together.

\section{Funding}

None to declare.

\section{Conflict of interest}

The authors have no conflicts of interest to declare.

\section{Author contributions}

Conception and design of the study (NM-S), data acquisition (AV-R, XQ, EMY, MR-G, JG, RT, LA, APK), writing of the manuscript, critical revision of the manuscript and approval of the final version to be published (NM-S, AV-R, XQ, EMY, MRG, JG, RT, LA, APK).

\section{References}

[1] Gentile I, Abenavoli L. COVID-19: Perspectives on the potential novel global threat. Rev Recent Clin Trials 2020. doi: 10.2174/1574887115999200228100745.

[2] Ashour HM, Elkhatib WF, Rahman MM, Elshabrawy HA. Insights into the recent 2019 novel coronavirus (SARS-CoV-2) in light of past human coronavirus outbreaks. Pathogens 2020;9. doi: 10.3390/pathogens9030186.

[3] Jia HP, Look DC, Shi L, Hickey M, Pewe L, Netland J, et al. ACE2 receptor expression and severe acute respiratory syndrome coronavirus infection 
depend on differentiation of human airway epithelia. J Virol 2005;79:1461414621. doi: 10.1128/JVI.79.23.14614-14621.2005.

[4] Cai H. Sex difference and smoking predisposition in patients with COVID-19. Lancet Respir Med 2020;8:e20. doi: 10.1016/S2213-2600(20)30117-X.

[5] World Health Organization. European tobacco use: Trends report 2019 (2019). Available from: http://www.euro.who.int/en/health-topics/diseaseprevention/tobacco/publications/2019/european-tobacco-use-trendsreport-2019-2019

[6] Doherty PC, Zinkernagel RM. A biological role for the major histocompatibility antigens. Lancet 1975;1:1406-1409. doi: 10.1016/s0140-6736(75)92610-0.

[7] Lin M, Tseng HK, Trejaut JA, Lee HL, Loo JH, Chu CC, et al. Association of HLA class I with severe acute respiratory syndrome coronavirus infection. BMC Med Genet 2003;4:9. doi: 10.1186/1471-2350-4-9.

[8] Ng MHL, Lau KM, Li L, Cheng SH, Chan WY, Hui PK, et al. Association of humanleukocyte-antigen class I (B*0703) and class II (DRB1*0301) genotypes with susceptibility and resistance to the development of severe acute respiratory syndrome. J Infect Dis 2004;190:515-518. doi: 10.1086/421523.

[9] Wang SF, Chen KH, Chen M, Li WY, Chen YJ, Tsao CH, et al. Human-leukocyte antigen class I CW 1502 and class II DR 0301 genotypes are associated with resistance to severe acute respiratory syndrome (SARS) infection. Viral Immunol 2011;24:421-426. doi: 10.1089/vim.2011.0024.

[10] Nguyen A, David JK, Maden SK, Wood MA, Weeder BR, Nellore A, et al. Human leukocyte antigen susceptibility map for SARS-CoV-2. medRxiv 2020. doi: 10.1101/2020.03.22.20040600.

[11] Miller A, Reandelar MJ, Fasciglione K, Roumenova V, Li Y, Otazu GH. Correlation between universal BCG vaccination policy and reduced morbidity and mortality for COVID-19: an epidemiological study. medRxiv 2020. doi: 10. 1101/2020.03.24.20042937.

[12] Chen N, Zhou M, Dong X, Qu J, Gong F, Han Y, et al. Epidemiological and clinical characteristics of 99 cases of 2019 novel coronavirus pneumonia in Wuhan, China: a descriptive study. Lancet 2020;395:507-513. doi: 10. 1016/S0140-6736(20)30211-7.

[13] Guan WJ, Ni ZY, Hu Y, Liang WH, Ou CQ, He JX, et al. Clinical characteristics of coronavirus disease 2019 in China. N Engl J Med 2020;382.doi: 10. 1056/NEJMc2005203.

[14] Wu YY, Li HY, Xu XB, Zheng KX, Qi XS, Guo XZ. Clinical characteristics and outcomes of 2019 novel coronavirus pneumonia: a meta-analysis (in Chinese). Chin J Hepatol 2020;28:240-246.
[15] Yao N, Wang SN, Lian JQ, Sun YT, Zhang GF, Kang WZ, et al. Clinical characteristics and influencing factors of patients with novel coronavirus pneumonia combined with liver injury in Shaanxi region (in Chinese). Zhonghua Gan Zang Bing Za Zhi 2020;28:E003. doi: 10.3760/cma.j.cn501113-2020022600070.

[16] The Chinese Digestion Association of the Chinese Medical Doctor Association, the Chinese Society of Hepatology of the Chinese Medical Association. The protocol for prevention, diagnosis and treatment of liver injury in coronavirus disease 2019 (in Chinese). Zhonghua Gan Zang Bing Za Zhi 2020; 28:218-221. doi: 10.3760/cma.j.cn501113-20200309-00095.

[17] Guan GW, Gao L, Wang JW, Wen XJ, Mao TH, Peng SW, et al. Exploring the mechanism of liver enzyme abnormalities in patients with novel coronavirusinfected pneumonia (in Chinese). Zhonghua Gan Zang Bing Za Zhi 2020;28: E002. doi: 10.3760/cma.j.issn.1007-3418.2020.02.002.

[18] Chau T, Lee KC, Yao H, Tsang TY, Chow TC, Yeung YC, et al. SARS-associated viral hepatitis caused by a novel coronavirus: Report of three cases. Hepatology 2004;39:302-310. doi: 10.1002/hep.20111.

[19] Hu LL, Wang WJ, Zhu QJ, Yang L. Novel coronavirus pneumonia related liver injury: etiological analysis and treatment strategy (in Chinese). Zhonghua Gan Zang Bing Za Zhi 2020;28:E001. doi: 10.3760/cma.j.issn.1007-3418. 2020.02.001.

[20] Huang Y, Gao Z. Study of the relationship SARS and hepatitis virus B (in Chinese). Chin J Clini Hepatol 2003:342-343.

[21] Wang FS. Current status and prospects of studies on human genetic alleles associated with hepatitis B virus infection. World J Gastroenterol 2003;9: 641-644. doi: 10.3748/wjg.v9.i4.641.

[22] Zhang C, Shi L, Wang FS. Liver injury in COVID-19: Management and challenges. Lancet Gastroenterol Hepatol 2020. doi: 10.1016/S2468-1253(20) 30057-1.

[23] D'Antiga L. Coronaviruses and immunosuppressed patients. The facts during the third epidemic. Liver Transpl 2020. doi: 10.1002/It.25756

[24] Qin J, Wang H, Qin X, Zhang P, Zhu L, Cai J, et al. Perioperative presentation of COVID-19 disease in a liver transplant recipient. Hepatology 2020. doi: 10.1002/hep.31257.

[25] Xiao Y, Pan H, She Q, Wang F, Chen M. Prevention of SARS-CoV-2 infection in patients with decompensated cirrhosis. Lancet Gastroenterol Hepatol 2020. doi: $10.1016 /$ S2468-1253(20)30080-7. 